\title{
Airfoil Design for Vertical Axis Wind Turbine Operating at Variable Tip Speed Ratios
}

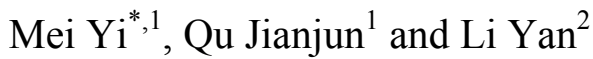 \\ ${ }^{1}$ School of Mechatronics Engineering, Harbin Institute of Technology, Harbin, Heilongjiang, 15001, China; ${ }^{2}$ School of \\ Engineering, Northeast Agricultural University, Harbin, Heilongjiang, 150030, China
}

\begin{abstract}
A new airfoil design method for $\mathrm{H}$ type vertical axis wind turbine is introduced in the present study. A novel indicator is defined to evaluate vertical axis wind turbine aerodynamic performance at variable tip speed ratios and selected as the airfoil design objective. A mathematic model describing the relationship between airfoil design variables and objective is presented for direct airfoil design on the basis of regression design theory. The aerodynamic performance simulation is conducted by computational fluid dynamics approach validated by a wind tunnel test in the study. Based on the newly developed mathematic model, a new airfoil is designed for a given wind turbine model under constant wind speed of $8 \mathrm{~m} / \mathrm{s}$. Meanwhile, the comparison of aerodynamic performance for newly designed airfoil and existing vertical axis wind turbine airfoils is studied. It has been demonstrated that, by the novel indicator, the rotor aerodynamic performance at variable tip speed ratios with the newly designed airfoil is $6.78 \%$ higher than the one with NACA0015 which is the airfoil widely used in commercial $\mathrm{H}$ type vertical axis wind turbine.
\end{abstract}

Keywords: Airfoil design, Computational fluid dyanmics, Variable tip speed ratios, Vertical axis wind turbine, Horizontal axis wind turbines, Wind turbine rotor model.

\section{INTRODUCTION}

There is a growing interest in harvesting wind energy within the built environment in urban areas with the increasing focus on off-grid energy generation [1-4]. Horizontal axis wind turbines (HAWTs) and vertical axis wind turbines (VAWTs) are two main types of equipment applied in converting the kinetic energy in the wind into mechanical energy. It has been found by several researchers, $\mathrm{H}$ type VAWTs are particularly well suited to residential wind power generation for some inherent advantages in comparing with their HAWT counterparts [5-7]. Those merits are: being able to extract energy independently from wind direction, lower production costs, quieter in operation, and ease of installation and maintenance.

To achieve better wind turbine aerodynamic performance, the selection of the airfoil plays a crucial role. For commercial $\mathrm{H}$ type VAWTs, symmetric airfoils from the NACA 4-digit series are commonly employed because only for these airfoils aerodynamic characteristics are the most well documented [8]. Due to the particular requirements, efforts have been made to improve the VAWT airfoil profile based upon the NACA 4-digit series in recent years. In 2006, Claessens [9] designed DU 06-W-200 based upon NACA 0018. The new airfoil has a much higher maximum lift coefficient for positive angles of attack, resulting in a wider drag bucket, deep stall occurring at higher angles of attack with a smaller drop in lift coefficient. One year later, Islam et al. [10] developed an airfoil named M1-VAWT1 with the

*Address correspondence to this author at the School of Mechatronics, Harbin Institute of Technology, Harbin, Heilongjiang, 150001, China; Tel: 0086-13654543250; E-mail: darrenymei@gmal.com purpose of ensuring the airfoil to generate more torque at low Reynolds number which is the operating condition of VAWT. In 2011, Qu et al. [11] designed an airfoil named OPTFoil for VAWT in attempt to make the blade obtain higher tangential force during the rotation. More recently, Shu et al. [12] presented a tailored airfoil on the basis of NACA 0012 and result shows that tailored airfoil exhibits better stall characteristics than NACA0012.

The existing research focused on improving the aerodynamic characteristics of single airfoil. However, an $\mathrm{H}$ type VAWT rotor is consisted of multiple blades, signifying that the improvement of single airfoil aerodynamic performance does not guarantee the perfectly better aerodynamic performance of the wind rotor. It is necessary to alter the design goal from individual airfoil to entire wind rotor. Moreover, when applied in urban sites, $H$ type VAWTs encounter the problem that operating at variable tip speed ratios, which is attributed to complex wording condition featured by the matching of rotor and generator, electric storage and consumption, changing of electrical load [13-15]. Thus, using a power coefficient at fixed tip speed ratio to assess rotor aerodynamic performance at variable tip speed ratios is confusing and an attempt to develop a new aerodynamic performance evaluation indicator should be made. On another aspect, CFD method coupled with optimization algorithm has been routinely applied in wind turbine airfoil design [16, 17]. It should not be neglected that the CFD calculation, though having relatively accurate prediction results compared to other aerodynamic calculating tools, tends to be time consuming when the study object is $\mathrm{H}$ type VAWT characterized with complex flow field. Then, how to decrease the computational cost becomes one of the major challenges during the airfoil design process. 
The present work is dedicated to design an airfoil for $\mathrm{H}$ type VAWTs applied in urban sites, contributing better rotor aerodynamic performance in comparison of existing VAWT airfoils. The research is conducted from two aspects: developing a new indicator in order to evaluate aerodynamic performance under a range of tip speed ratios meanwhile selecting this indicator as airfoil design objective, and constructing a mathematic model describing the explicit functional relationship between airfoil design variables and objective for direct airfoil design, looking to reduce computational time in airfoil design process.

\section{THE WIND TURBINE ROTOR MODEL}

It has been suggested by reference [18] that the value of height to radius ratio for VAWT applied in urban areas is between 2 to 3. Also, a three-bladed VAWT rotor is structurally non-directional and tends to run smoothly because of lower fluctuations of energy in each revolution [19]. Assuming the wind speed in urban regions is usually between $6 \mathrm{~m} / \mathrm{s}$ to $10 \mathrm{~m} / \mathrm{s}$ [20], the wind velocity value in this study was maintained at $8 \mathrm{~m} / \mathrm{s}$. Major geometry specifications of scaled VAWT rotor are categorized and listed in Table $\mathbf{1 .}$
Table 1. Major rotor geometry specifications.

\begin{tabular}{|c|c|}
\hline Parameter & Value \\
\hline \hline Rotor radius, $(\mathrm{m})$ & 0.2 \\
\hline Rotor central shaft radius, $(\mathrm{m})$ & 0.01 \\
\hline Blade cord length, $(\mathrm{m})$ & 0.07 \\
\hline Blade length, $(\mathrm{m})$ & 0.5 \\
\hline Number of blade & 3 \\
\hline
\end{tabular}

\section{METHOD FOR AIRFOIL DESIGN}

The rotor aerodynamic performance at variable tip speed ratios is selected as the airfoil design objective. A new indicator named averaged effective wind energy utilization coefficient has been developed as represented in section 3.1. Airfoil geometry generation which allows the translation of the variables into the actual airfoil is an important part of the airfoil design, and the geometry generation method is described in section 3.2. This paper aims to build a mathematic model for direct airfoil design on the basis of relatively small amount of CFD calculation by quadratic

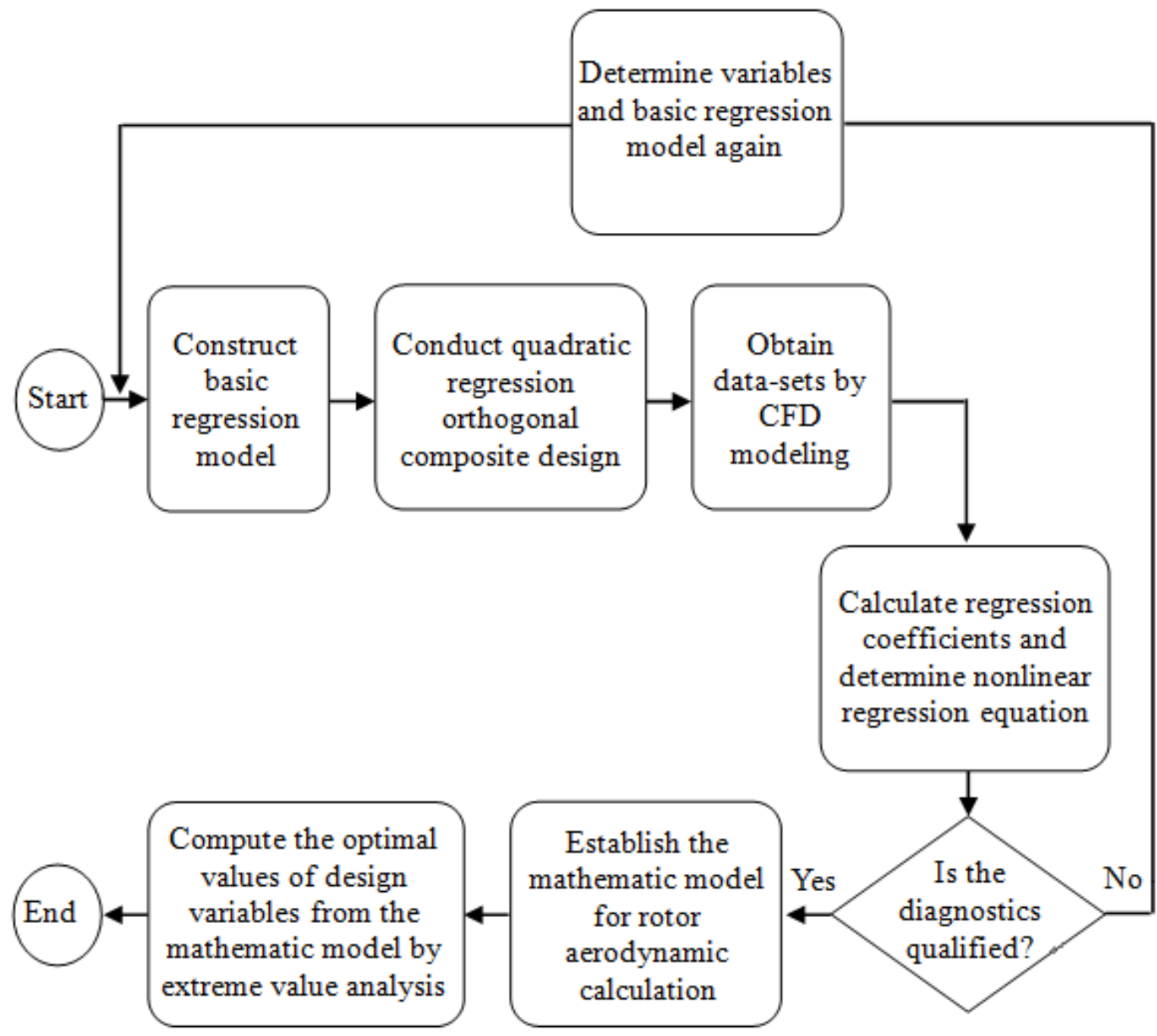

Fig. (1). Flow chart of airfoil design process. 
regression orthogonal composite design, which is illustrated in section 3.3. Also, details of CFD approach are stated in section 3.4. Fig. (1) shows the flow chart of airfoil design process.

\subsection{Design Objective Analysis}

It is widely acknowledged that the wind turbine harness the kinetic energy contained in flowing air masses. The wind turbine firstly transforms the power available in wind to the power output from the rotor. Then the mechanical energy is transformed to the electronic power by the generator. It is physically impossible to technically exploit the entire wind energy, so there is efficiency during the wind power transformed to mechanical power. Power coefficient $C_{P}$ written as Eq. (1) is an expression of turbine harness wind energy, and torque coefficient $C_{T}$ described by Eq. (2) reveals the torque performance of the rotor.

$C_{P}=\frac{2 T_{a v} \omega}{\rho S V_{\infty}^{3}}$

where $T_{\text {ave }}$ is the averaged torque of wind rotor during each revolution, $\omega$ is the rotor rotating angular velocity, $\rho$ is the density of the air, $S$ is the swept area of wind turbine and $V_{\infty}$ is the inlet air velocity.

$$
C_{T}=\frac{2 T_{\text {ave }}}{\rho S R V_{\infty}^{2}}
$$

The power coefficient reflects the aerodynamic performance of rotor. To evaluate the efficiency of rotor extracting wind power, $C_{P}$ of a fixed tip speed ratio is confusing. If the tip speed ratio $\lambda$ alters during the turbine operation, the power coefficient changes accordingly. An $\mathrm{H}$ type VAWT is supposed to work at wind speed of $V_{\infty}$ between time range $\left[t_{a}, t_{b}\right]$, which means the generator and electrical equipment are on regular operation and the output power is effective. The function of tip speed ratio $\lambda$ varies with time is denoted as $\lambda(t)$, and the range of $\lambda$ is $\left[\lambda_{a}, \lambda_{b}\right]$. Then the effective wind energy utilization coefficient $E_{e}$ in $\left[t_{a}, t_{b}\right]$ can be defined by the ratio of the extracted wind energy on the rotor swept area and the energy provided by the flowing air. The mathematical expression of $E_{e}$ is shown as Eq. (3).

$$
E_{e}=\frac{\frac{1}{2} \rho S V_{\infty}^{3} \int_{t_{a}}^{t_{b}} C_{P}[\lambda(t)] d t}{\frac{1}{2} \rho S V_{\infty}{ }^{3}}=\int_{t_{a}}^{t_{b}} C_{P}[\lambda(t)] d t
$$

However, it is not easy to provide the specific expression of $\lambda(t)$, as the complex reason of rotor operating at variable tip speed ratio. As an equipment harnessing energy from the wind, the evaluation of a rotor aerodynamic performance can be converted to estimate the overall potential of rotor absorbing effective wind energy resources. Thus, if the effective working range of $\lambda$ is $\left[\lambda_{a}, \lambda_{b}\right]$ under the wind velocity of $V_{\infty}$, regardless of the specific values of $\lambda$ at any time, the rotor aerodynamic performance can be defined by averaged effective wind energy utilization coefficient $E_{e a}$, as shown in Eq. (4).

$$
E_{e a}=\frac{1}{\lambda_{b}-\lambda_{a}} \int_{t_{a}}^{t_{b}} C_{P}(\lambda) d \lambda
$$

The power performance coefficient values obtained by numerical simulation are discrete data corresponding to tip speed ratios. Besides the step size of $\lambda$ is easy to maintain stable in the calculation, numerical integration can be applied in solving Eq. (4). Supposing the number of tip speed ratio data achieved in numerical simulation is $m$, the computing formula of $E_{\text {ea }}$ can be written as Eq. (5). Lift type VAWTs generate effective power when $\lambda>1$. Also, the noise emission level exceeds $60 \mathrm{~dB}$ when $\lambda>3$, according to Lida's study [21]. Thus, the range of $\lambda$ should be $[1,3]$ in practical calculation of $E_{e a}$.

$$
E_{e a}=\frac{1}{2(m-1)}\left[C_{p}\left(\lambda_{a}\right)+C_{p}\left(\lambda_{b}\right)+2 \sum_{i=1}^{m-2} C_{p}\left(\lambda_{i}\right)\right]
$$

\subsection{Airfoil Geometry Generation}

According to previous investigation [22, 23], thickness and camber are two parameters having major influence on rotor aerodynamic performance, which should be selected as design variables. Airfoil geometry generation is that one allows the translation of the design variables into the actual airfoil. The present work is on the basis of NACA 4-digit series airfoil which has been utilized by the majority of VAWTs. Reference [24] gives their shapes based upon rectangular coordinate system are given by the following equations:

$y_{\text {camber }}=\left\{\begin{array}{l}\frac{c_{\max }}{\left(1-x_{c \max }\right)^{2}}\left[\left(1-2 x_{c \max }\right)+2 x_{c \max } x-x^{2}\right. \\ \frac{c_{\max }}{x_{c \max }^{2}}\left(2 x_{c \max } x-x^{2}\right)\end{array}\right.$

where $y_{\text {camber }}$ is the $y$ coordinate of the mean camber line, $c_{\max }$ is the maximum camber value, $x_{\text {cmax }}$ is the $x$ coordinate of the maximum camber.

$$
\begin{aligned}
& \pm y_{\text {thick }}=5 t_{\text {max }}(0.2969 \sqrt{x}-0.126 x \\
& \left.-0.3516 x^{2}+0.2843 x^{3}-0.1015 x^{4}\right)
\end{aligned} \quad(0<x<1)
$$

where $y_{\text {thick }}$ is the $y$ coordinate of the thickness distribution, $t_{\max }$ is the maximum thickness value.

\subsection{Mathematic Model for Rotor Aerodynamic Computation}

Mathematic model developed in this part is used to describe the function relationship between rotor aerodynamic performance at variable tip speed ratios and the airfoil design variables. A second order nonlinear regression method is applied to build the model by calculation of data points in conjunction with composite design [25]. The relationship can be written in a general form as follows:

$$
y=\beta_{0}+\sum_{j=1}^{p} \beta_{j} x_{j}+\sum_{k<j} \beta_{j k} x_{k} x_{j}+\sum_{j=1}^{p} \beta_{j j} x_{j}^{2}
$$


where $y$ is the design objective, $x_{k}$ and $x_{j}$ are design variables, $p$ is the number of variables, $\beta_{0}, \beta_{j}, \beta_{j k}, \beta_{j j}$ are regression coefficients.

When applying composite design to obtain experiment data points, in order to ensure the orthogonality of experiment plan, Eq. (8) needs to be transformed into the pattern in the code space as shown in Eq. (9). The encoding formula is given by Eq. (10). The structure matrix and undetermined coefficients of Eq. (9) are given by Eq. (11) and Eq. (12).

$$
\begin{aligned}
& y=b_{0}+\sum_{j=1}^{p} b_{j} z_{j}+\sum_{k<j} b_{j k} z_{k} z_{j}+\sum_{j=1}^{p} b_{j j} z_{j}^{2} \\
& z_{j k}=\frac{x_{k j}-x_{0 j}}{\Delta_{j}} \quad(k=0,1,2 ; j=1,2, \ldots, p)
\end{aligned}
$$

where $x_{0 j}=\left(x_{1 j}+x_{2 j}\right) / 2$ and $\Delta_{j}=\left(x_{2 j}-x_{1 j}\right) / 2, x_{2 j}$ and $x_{1 j}$ are the upper and lower limit of $x_{j}$, separately.

$Z=\left[\begin{array}{cccccc}z_{10} & z_{11} & z_{12} & z_{11} z_{12} & z_{11}^{2} & z_{12}^{2} \\ z_{20} & z_{21} & z_{22} & z_{21} z_{22} & z_{21}^{2} & z_{22}^{2} \\ \vdots & \vdots & \vdots & \vdots & \vdots & \vdots \\ z_{110} & z_{111} & z_{112} & z_{111} z_{112} & z_{111}^{2} & z_{112}^{2}\end{array}\right]$

$$
\left\{\begin{array}{l}
b_{0}=\sum_{i=1}^{u} y_{i} / u \\
b_{j}=\sum_{i=1}^{u} z_{i j} y_{i} / \sum_{i=1}^{u} z_{i j}^{2} \\
b_{k j}=\sum_{i=1}^{u} z_{i k} z_{i j} y_{i} / \sum_{i=1}^{u}\left(z_{i k} z_{i j}\right)^{2} \\
b_{j j}=\sum_{i=1}^{u} z_{i j}^{\prime} y_{i} / \sum_{i=1}^{u} z_{i j}^{\prime 2}
\end{array}\right.
$$

where $u$ is the number of experiment.

Once the form of Eq. (9) is confirmed, it is necessary to process regression diagnostics where F-test is applied. Regression diagnostics is consisted of three parts, namely significance test on regression coefficients, significance test on regression equation and lack of fit test on regression model. The formula of significance test on regression coefficients is written as Eq. (13). If $F_{j}>F_{\alpha}\left(f_{j}, f_{e 2}\right), F_{j k}>F_{\alpha}\left(f_{j k}\right.$, $\left.f_{e 2}\right)$ and $F_{j j}>F_{\alpha}\left(f_{j j}, f_{e 2}\right)$, the design variables are significant under level $\alpha$, or the design variables need to be determined again.

$$
\left\{\begin{array}{l}
F_{j}=\frac{S_{j} / f_{j}}{S_{e 2} / f_{e 2}} \\
F_{k j}=\frac{S_{k j} / f_{k j}}{S_{e 2} / f_{e 2}} \\
F_{j j}=\frac{S_{j j} / f_{j j}}{S_{e 2} / f_{e 2}}
\end{array}\right.
$$

where $S_{e 2}$ is the residual sum of squares, $S_{j}, S_{j k}$ and $S_{j j}$ represent the linear term, interaction term and quadratic term sum of squares, $f_{e 2}$ is the additional freedom, $f_{j}, f_{j k}$ and $f_{j k}$ represent the degree of freedom for linear term, interaction term and quadratic term.

The significance test on regression coefficients is expressed by Eq. (14). If $F_{E}>F_{\alpha}\left(f_{E}, f_{e 2}\right)$, the regression equation is significant under level $\alpha$.

$F_{E}=\frac{S_{E} / f_{E}}{S_{e 2} / f_{e 2}}$

where $S_{E}$ is the regression sum of squares, $f_{E}$ is the degree of freedom for regression.

The test for lack of fit on regression model is conducted by Eq. (15), if $F_{l f}<F_{\alpha}\left(f_{l f}, f_{e}\right)$, the fit is good. Otherwise, there can be higher order relation between the design objective and variables, the regression model reevaluation is needed.

$F_{l f}=\frac{S_{l f} / f_{l f}}{S_{e} / f_{e}}$

where $S_{l f}$ is the sum of squares for lack of fit, $f_{l f}$ is the residual degree of freedom, $S_{e}$ is the error sum of squares for zero repetition test, $f_{e}$ is the degree of freedom for zero repetition test.

After the result of regression diagnostics is confirmed to be positive, Eq. (10) is applied again on Eq. (9) with all determined coefficients to obtain Eq. (8). Then, applying extreme value analysis method on Eq. (8) can resolve the optimal values of design variables and the geometry of the new airfoil is achieved.

\subsection{CFD Approach for Aerodynamic Simulation}

The commercial CFD package was used for the aerodynamic simulations. The code uses the finite volume method to solve the governing equations for fluids. The incompressible unsteady Reynolds Averaged Navier-Stokes equations were solved for the rotor flow field. The coupled pressure based solver was selected with a second order implicit transient formulation for improved accuracy. The PISO algorithm was applied for the solution of the pressurevelocity coupled equations. All solution variables were solved via QUICK scheme which is a high order up wind method in view of the complexity of the flow. All the residuals are set to $10^{-5}$ for all the variables. The shear stress transport (SST) k- $\omega$ model [26] has been successfully applied for many numerical simulations of $H$ type VAWT [27-29]. Therefore, the SST k- $\omega$ model was employed for all the simulations presented in this paper.

The geometry of the rotor was created by using Gambit software. Fig. (2) is the $2 \mathrm{D}$ schematic of the computational domain employed for computations, where $\Phi$ is noted as the diameter of the rotor. The domain is created by assembling a circular rotating region in a rectangular stationary region $\mathrm{ABCD}$ where the rectangular region is $20 \Phi$ in length and $10 \Phi$ in width, and the circular rotating region has a diameter of $2 \Phi$. For the rectangular domain, AC side is set as an inlet boundary condition whereas BD side set as a pressure outlet boundary with six no slip boundaries to three blade surfaces, rotor central shaft, $\mathrm{AB}$ and $\mathrm{CD}$ sides. The boundary where rectangular region connects to circular rotating region is set as an interface boundary condition in order to employ the 
sliding mesh technique. According to the rotational speed of the rotor, the time for an equivalent of half degree rotation of the rotor was adopted for numerical time step size.

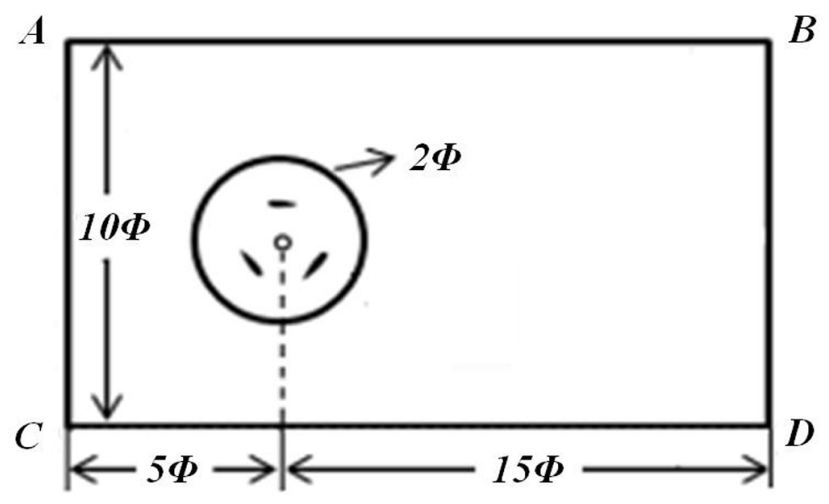

Fig. (2). 2D schematic of the computational domain.

Structured quadrilateral grids were used for the domain meshing. As shown by Fig. (3a), O type mesh was adapted around each blade. The height of the first cell used was $1.07 \times 10^{-4} \mathrm{~m}$ and the growth rate of inflation was implemented as 1.07 to give an average of the y plus values from the flow solutions of 5 to 10. Fig. (3b) shows the mesh in the domain. To reduce computation time, the stationary domain was relatively coarsely meshed with a maximum edge length of the cells not exceeding a blade cord length. A mesh independent test was also performed under one rotor tip speed ratio. Several different structured grids composed of variable mesh size of the entire domain ranging from 100,000 to 700,000 cells have been examined. It has been determined that around a mesh number of 600,000 cells on the domain was the level at which grid independence was attained.

(a)

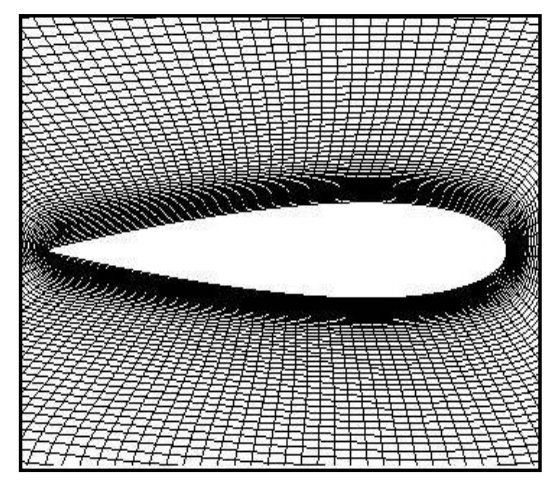

(b)

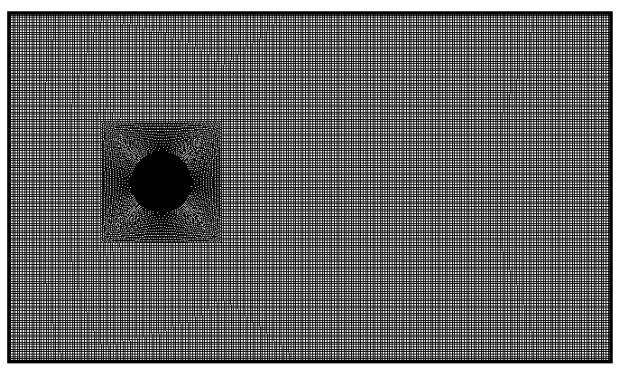

Fig. (3). Mesh generation: (a) mesh around the airfoil; (b) mesh in the domain.
To ensure a full development of the flow, several revolutions have to be simulated before converged periodical solution is achieved. Therefore, when the relative difference of periodic averaged power coefficient over one revolution is less than $1 \%$, the solution is considered to be converged in this paper. As averaged power coefficient is related to averaged torque, the relative difference of averaged torque, $r$, is

$r=\left|\frac{T_{\text {ave }}(i+1)-T_{\text {ave }}(i)}{T_{\text {ave }}(i)}\right|<1 \%$

where $T_{\text {ave }}(i)$ is the averaged torque at rotor cycle number of $i$.

Instantaneous torque values can be calculated by Fluent during the simulation process, this makes torque be a function of time. Thus, the averaged torque $T_{\text {ave }}$ during the time interval $\left[t_{0}, t_{n}\right]$ is calculated by

$T_{\text {ave }}=\frac{1}{t_{n}-t_{0}} \int_{t_{0}}^{t_{n}} T(t) d t$

where $t_{0}$ represents the time at the beginning of a single rotation of the rotor and $t_{n}$ is the time at the end of a single rotation.

As instantaneous torque values are recorded in terms of discrete data by Fluent, by applying Newtown-Stokes formulas, Eq. (17) can be written as

$T_{\text {ave }}=\frac{\Delta t}{2\left(t_{n}-t_{0}\right)}\left[T\left(t_{0}\right)+2 \sum_{i=1}^{\frac{t_{n}-t_{0}}{\Delta t}-1} T\left(t_{i}\right)+T\left(t_{n}\right)\right]$

where $\Delta t$ is the numerical time step size value, $T\left(t_{i}\right)$ is the computed instantaneous torque of rotor at $t_{i}$.

Based on the numerical results of instantaneous torque, the computed values rotor power coefficient at every individual tip speed ratio can be obtained by combining Eq. (18) and Eq. (1).

\section{VALIDATION OF CFD SIMULAITON}

The CFD simulation should be checked against experimental data to assess its capability of correctly simulating VAWT aerodynamic performance. The experimental test was conducted in a low speed wind tunnel with an outlet of $1 \mathrm{~m} \times 1 \mathrm{~m}$. This tunnel is an open jet type with total length of $13 \mathrm{~m}$, including power section, diffuser, stilling chamber and contract section. The wind is generated from an axial fan driven by an inverter controlled electric motor, allowing a range of air velocity from $1 \mathrm{~m} / \mathrm{s}$ to $20 \mathrm{~m} / \mathrm{s}$, with a value of $1 \%$ turbulence intensity. An experimental platform is settled in front of the contraction. Fig. (4) shows the view of assembled wind turbine test platform in front of the wind tunnel. The major geometric values of the wind rotor and profile of the blade are consistent with the data listed in Table 1. The height of the foundation has been well designed to ensure that the wind rotor is positioned in the middle of the wind tunnel outlet. 


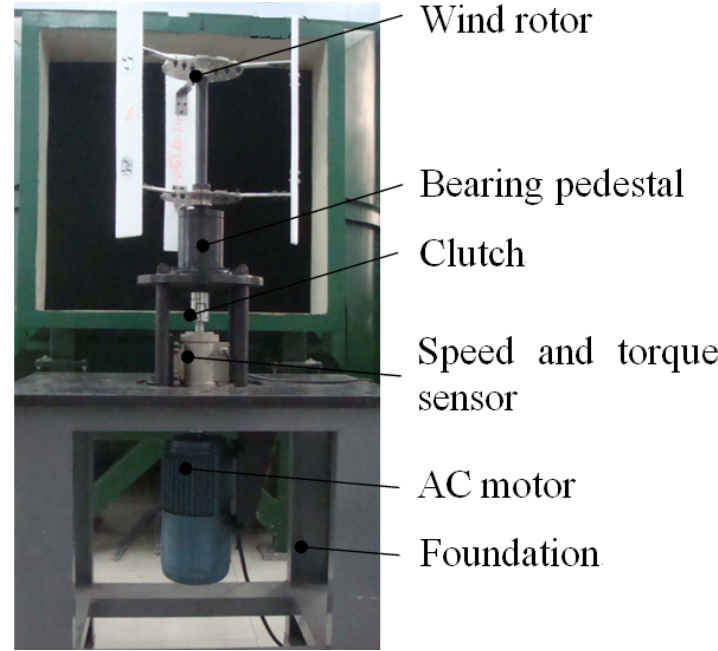

Fig. (4). Assembled platform.

The comparison between the CFD results and the experimental data of power coefficient at the constant wind speed of $8 \mathrm{~m} / \mathrm{s}$ is illustrated by Fig. (5). As shown in the figure, simulation results are appeared to be comparatively well matched together with the experimental data. However, some deviations are still observed and the averaged relative error is $14.08 \%$. As far as absolute power coefficient value is concerned, the validation is not that exact. This is because the CFD model is $2 \mathrm{D}$ while the actual problem is $3 \mathrm{D}$. Nevertheless, it is not wisely to apply 3D CFD simulation in the airfoil design because 3D simulation is extraordinary time consuming for VAWT. Moreover, the purpose of applying numerical simulation in airfoil design is progressing comparative analysis of aerodynamic performance, meaning absolute levels of performance are not important and only relative values of performance are needed. Considering the same tendency of two power performance curves, especially the good agreement in optimum tip speed ratio prediction, the CFD method conducted in this study is capable to capture the flow physics and suitable for aerodynamic computation in airfoil design.

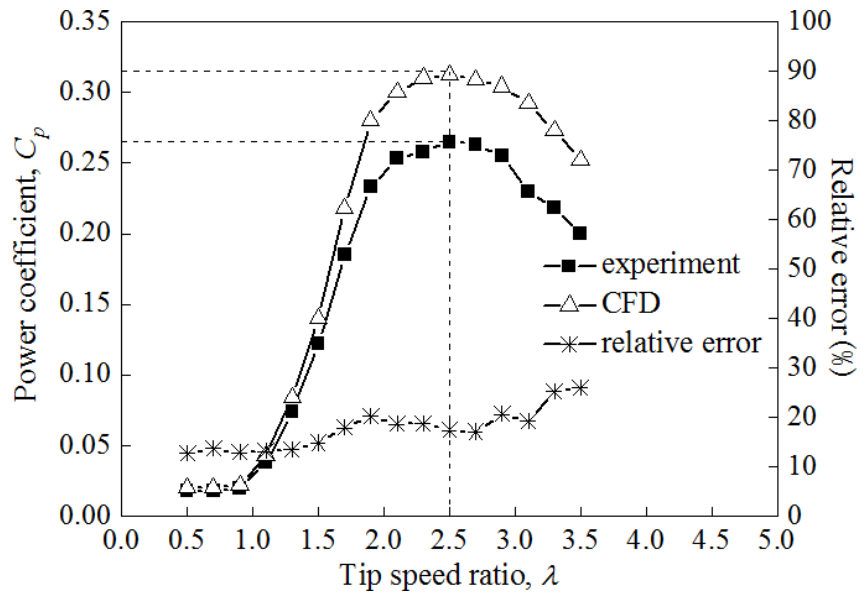

Fig. (5). Comparison of power coefficients obtained by different methods.

\section{DESIGN RESULTS AND ANALYSIS}

According to Eq. (6), five airfoils with different thickness can be obtained by controlling values of $t_{\max }$ at $12 \%, 15 \%$, $18 \%, 20 \%$ and $22 \%$, noted as T12, T15, T18, T20 and T22, separately. According to Eq. (7), five airfoils with different camber can be obtained by controlling values of $c_{\max }$ at 0 , $1 \%, 2 \%, 3 \%$ and $4 \%$, noted as $\mathrm{C} 0, \mathrm{C} 1, \mathrm{C} 2, \mathrm{C} 3$ and $\mathrm{C} 4$, separately. By CFD simulation, trends of averaged effective wind energy utilization coefficient $E_{e a}$ dependent on different thickness and camber values are illustrated in Figs. $(6,7)$. It can be seen from Fig. (6), $E_{\text {ea }}$ increases with the rising $t_{\max }$ and the maximum value attains at $t_{\max }=18 \%$ before decreasing to 0.235 at $t_{\max }=20 \%$ by $3.69 \%$. The figure shows a further $11.48 \%$ drop when $t_{\max }$ reaches $22 \%$. Similar trends can be found in Fig. (7), the maximum $E_{e a}$ achieves at $c_{\max }=1 \%$, but further rising of $c_{\max }$ leads to a decline of $E_{e a}$. The decreasing rate is $3.7 \%$ when the value of $c_{\max }$ alters from $1 \%$ to $4 \%$. It can be concluded from those analysis that there is a sharp decline of $E_{e a}$ when $t_{\max }$ exceeds $20 \%$, and the amplitude range of $E_{e a}$ is smaller when $t_{\max } \in[0,4 \%]$. Therefore, the design constraint conditions are as follows: $15 \% \leq t_{\max } \leq 20 \%, 0 \leq c_{\max } \leq 4 \%$.

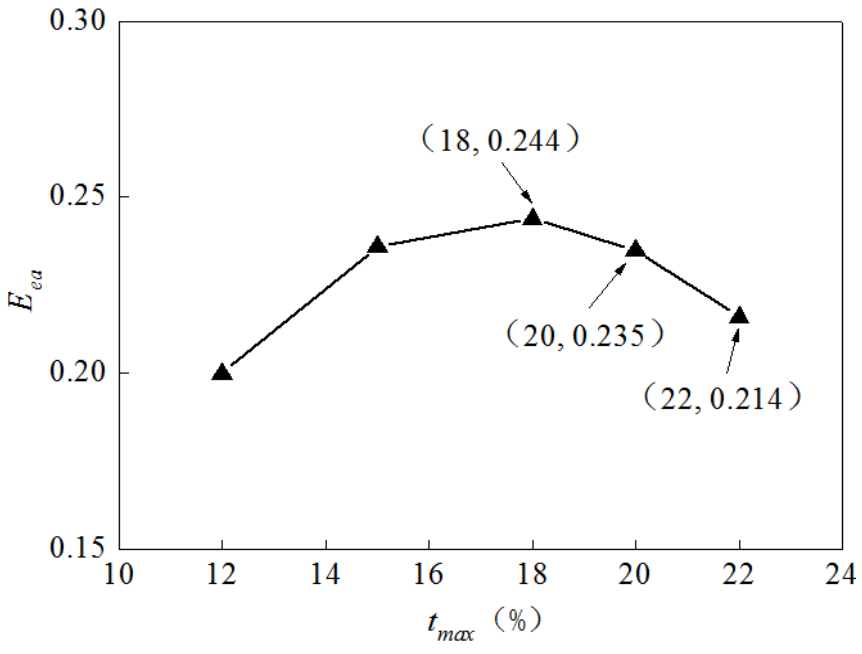

Fig. (6). $E_{\text {ea }}$ dependent on different $t_{\max }$ values.

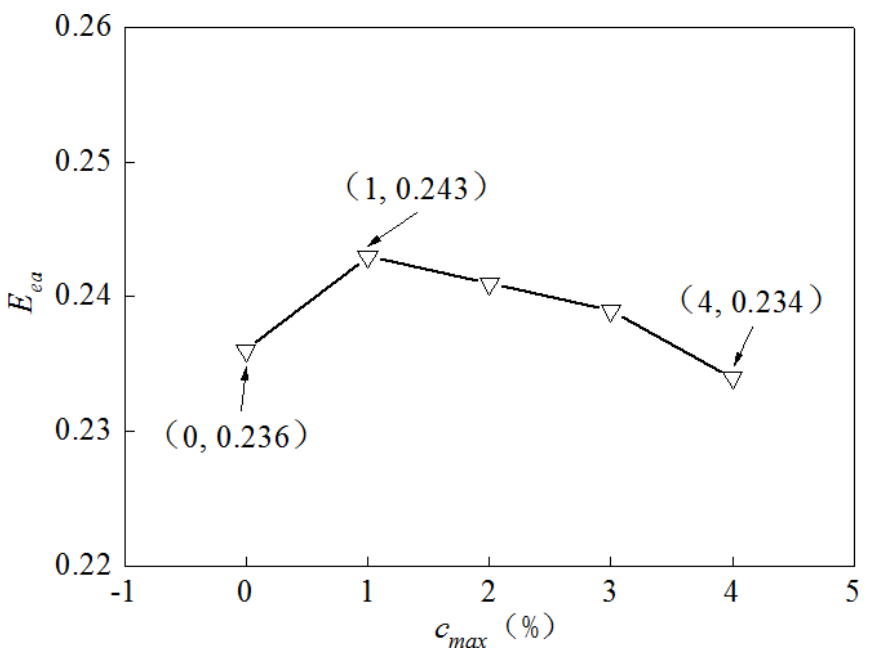

Fig. (7). $E_{e a}$ dependent on different $c_{\max }$ values. 
Assuming $t_{\max }=x_{1}$ and $c_{\max }=x_{2}$ and applying Eq. (10), the following exists: $x_{01}=0.175, \quad x_{02}=0.02, \quad \Delta_{1}=0.02178$, $\Delta_{2}=0.01742$. The experimental plan, including three zero repetition tests, is shown by Table 2 .

Table 2. Experimental plan.

\begin{tabular}{|c|c|c|}
\hline No. & $\mathbf{x 1}$ & $\mathbf{x 2}$ \\
\hline \hline 1 & 0.15322 & 0.00258 \\
\hline 2 & 0.15322 & 0.03742 \\
\hline 3 & 0.19678 & 0.00258 \\
\hline 4 & 0.19678 & 0.03742 \\
\hline 5 & 0.15 & 0.02 \\
\hline 6 & 0.2 & 0.02 \\
\hline 7 & 0.175 & 0 \\
\hline 8 & 0.175 & 0.04 \\
\hline 9 & 0.175 & 0.02 \\
\hline 10 & 0.175 & 0.02 \\
\hline 11 & 0.175 & 0.02 \\
\hline
\end{tabular}

Having obtained experimental data points by CFD approach and applying Eq. (9) to Eq. (14), the mathematic calculating model of rotor aerodynamic performance at variable tip speed ratios can be written as Eq. (19). Through extreme value analysis of Eq. (19), the maximum $E_{\text {ea }}$ obtained at $t_{\max }=17.45 \%$ and $c_{\max }=1.98 \%$. The newly designed airfoil is noted as OPTFoil2014. Fig. (8) shows the comparison of airfoil configuration between OPTFoil2014 and widely used VAWT airfoil NACA0015.

$$
\begin{aligned}
E_{e a}= & -0.1674+4.7 t_{\max }-13.43 t_{\max }^{2} \\
& +0.74 c_{\max }-18.49 c_{\max }^{2}
\end{aligned}
$$

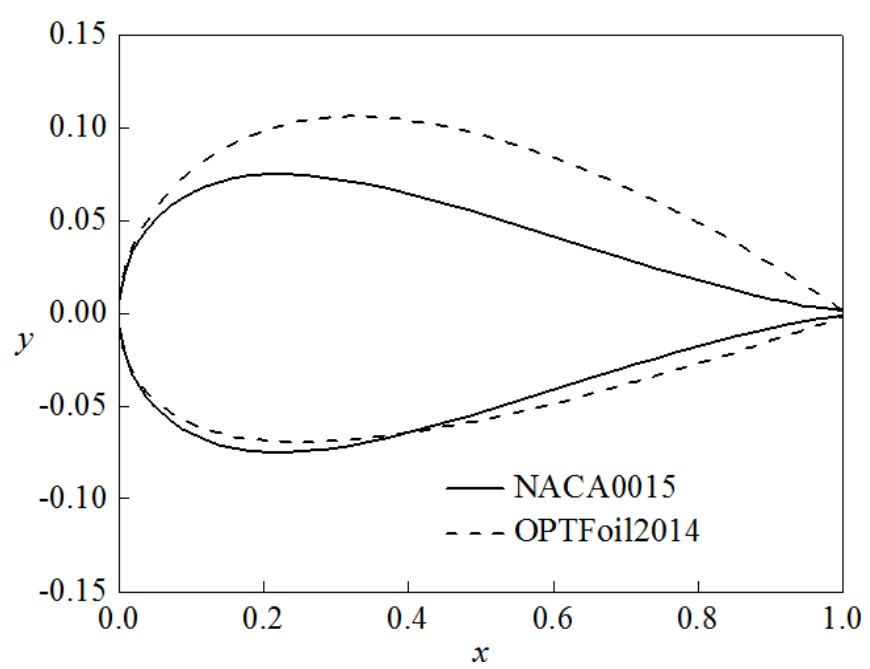

Fig. (8). Airfoil configurations of OPTFoil2014 and NACA0015.

Table 3 shows the statistics of calculated averaged effective wind energy utilization coefficients of $H$ type VAWT rotors with the four different airfoils, namely NACA0015, DU 06-W-200, OPTFoil and OPTFoil2014, among which, NACA0015 is the widely used airfoil for commercial VAWT, DU 06-W-200 and OPTFoil are foils designed with the design objective of improving single airfoil aerodynamic performance, and OPTFoil2014 is the newly designed foil with the design objective of improving rotor aerodynamic performance at variable tip speed ratios. It is seen from the table, compared to rotor applied NACA0015, values of $E_{e a}$ obtained by rotors with DU $06-W-$ 200, OPTFoil and OPTFoil2014 are higher and the maximum value of 0.252 is achieved by OPTFoil2014. By using OPTFoil2014, $E_{e a}$ of rotor can increase by $6.78 \%$ in comparison of those with NACA0015.

Table 3. Effective wind energy utilization coefficients of rotors with four different airfoils.

\begin{tabular}{|c|c|}
\hline Airfoil & $\boldsymbol{E}_{\text {ea }}$ \\
\hline \hline NACA0015 & 0.236 \\
\hline DU 06-W-200 & 0.244 \\
\hline OPTFoil & 0.238 \\
\hline OPTFoil2014 & 0.252 \\
\hline
\end{tabular}

Power performance of rotors with various airfoils is given by Fig. (9). It can be seen from the figure, rotor with DU 06-W-200 has higher power coefficient than others at the region of $1<\lambda<2$, while rotors with NACA0015 and OPTFoil2014 maintain high power performance at the region of $2<\lambda<3$. To have a better understanding of the differences, instantaneous torque coefficient $\left(C_{\text {Tinst }}\right)$ curves and flow field of rotors with various airfoils are analyzed at $\lambda=1.1$ and $\lambda=2.9$ (corresponding to positions A and B as signed in Fig. 11) which are selected as representatives of lower and higher tip speed ratios.

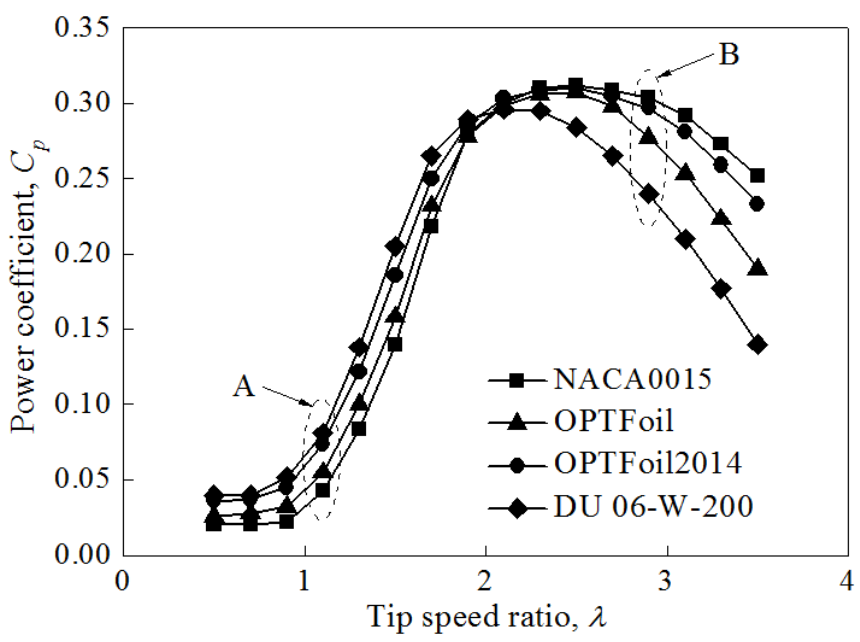

Fig. (9). Power performance of rotors with four different airfoils.

Figs. $(\mathbf{1 0}, \mathbf{1 1})$ show the instantaneous torque performance and vortex contours at $\lambda=1.1$ and $\lambda=2.9$, separately. As seen in Fig. (10a), all instantaneous torque coefficient curves attain the peak values at $\theta=60^{\circ}, \theta=180^{\circ}$ and $\theta=300^{\circ}$, while fall to the bottom at $\theta=0^{\circ}, \theta=120^{\circ}$ and $\theta=240^{\circ}$. The major difference of these curves exits in the process $C_{\text {Tinst }}$ dropping from the peak to bottom, where $C_{\text {Tinst }}$ of rotor with 
(a)

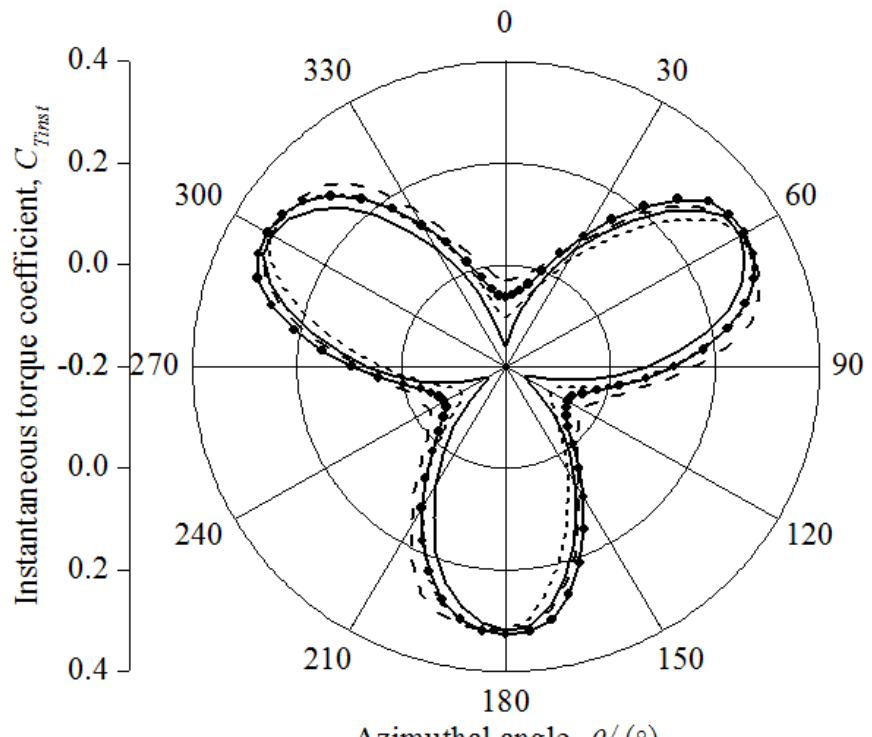

Azimuthal angle, $\theta /\left(^{\circ}\right)$

-NACA0015 - - - DU 06-W-200

(b)

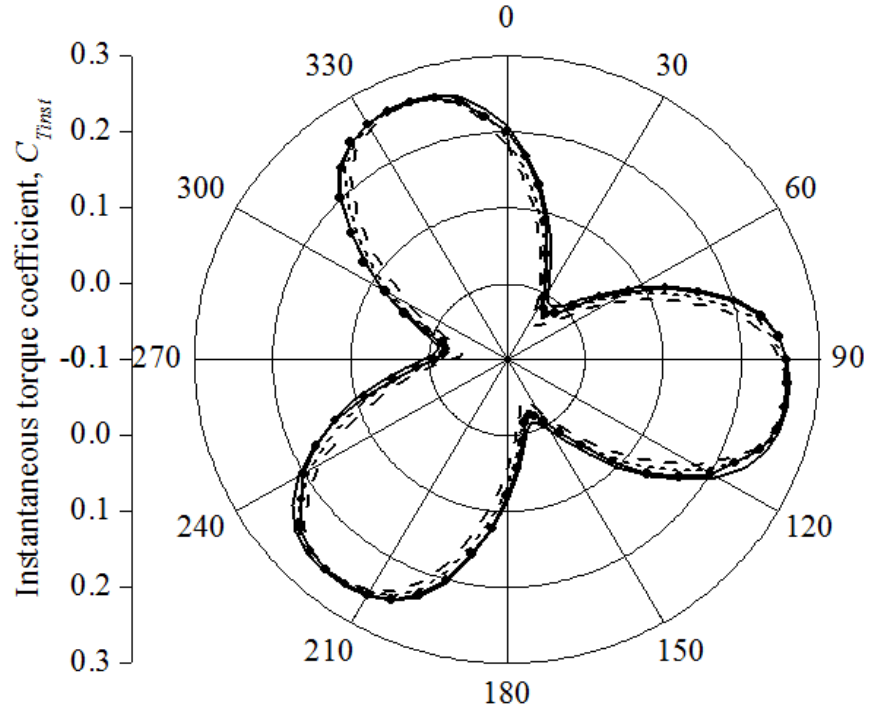

Azimuthal angle, $\theta /\left(^{\circ}\right)$

OPTFoil

OPTFoil2014

Fig. (10). Instantaneous torque performance of rotors with different airfoils at $\lambda=1.1$ and $\lambda=2.9$ : (a) $\lambda=1.1$; (b) $\lambda=2.9$.

NACA0015 drops faster than others and the slowest decrease comes from the rotor with DU 06-W-200. Flow physics is shown in Fig. (11a) which is featured by dynamic stall and flow separation. Both vortex shedding and flow separate can weaken the aerodynamic performance of blade. For blade 1, flow separation moving toward leading edge can be observed at NACA0015 and OPTFoil, trailing edge separation is seen on OPTFoil2014, and no sign of separation can be found on DU 06-W-200. For blade 2, vortexes have been separated from the blades of NACA0015 and OPTFoil. But the scale of shedding vortex from NACA0015 is larger than the one from OPTFoil, leading to a lower aerodynamic performance. When it comes to DU 06-W-200 and OPTFoil2014, the (a)

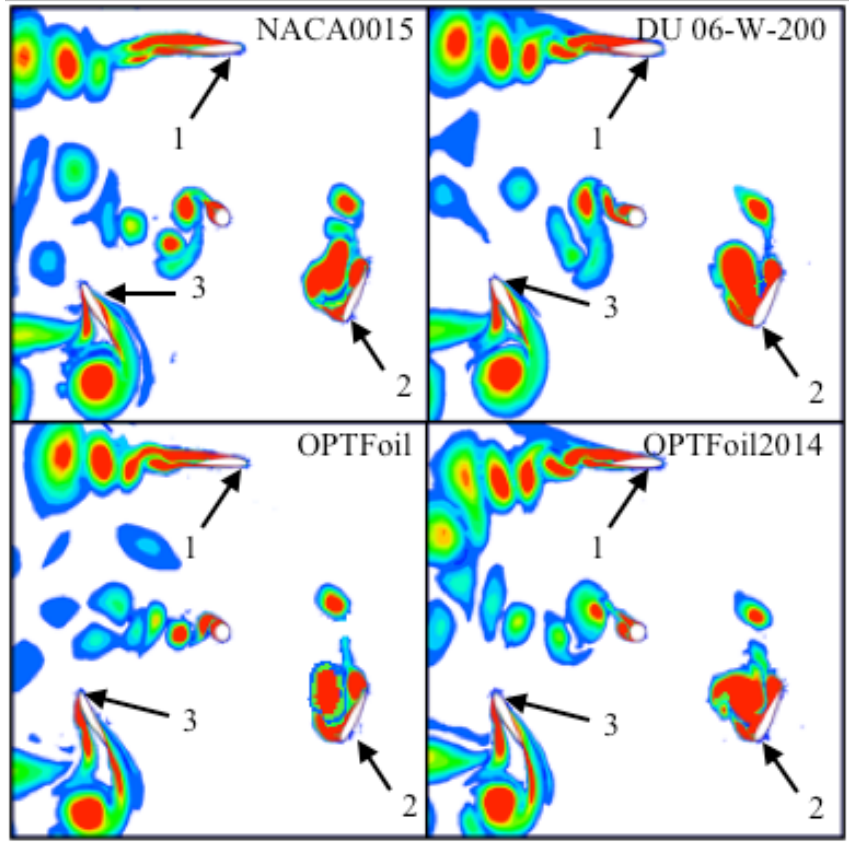

(b)

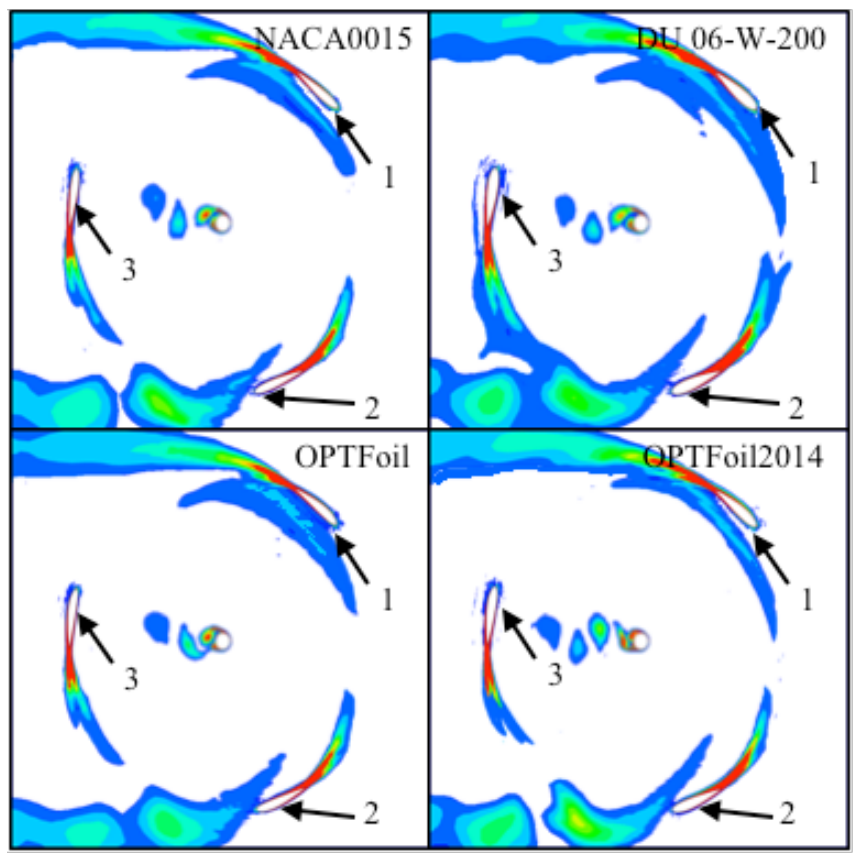

Vorticity (1/s) 50100150200250300350400450500

Fig. (11). Vortex contours of rotors with different airfoils at $\lambda=1.1$ and $\lambda=2.9$ : (a) $\lambda=1.1$; (b) $\lambda=2.9$.

vortex is still developing and about to shed, leading to a higher aerodynamic performance. Flow separation and vortex shedding at DU 06-W-200 and OPTFoil2014 are delayed during the rotor rotating compared with NACA0015 and OPTFoil, resulting in relatively higher torque coefficient and power coefficient at lower tip speed ratio. As seen in Fig. (10b), all instantaneous torque coefficient curves reach the peak values at $\theta=100^{\circ}, \theta=220^{\circ}$ and $\theta=340^{\circ}$, while fall to 
the bottom at $\theta=40^{\circ}, \theta=160^{\circ}$ and $\theta=280^{\circ}$. The major difference of these curves also exits when $C_{\text {Tinst }}$ dropping from the peak to bottom. It can be observed from Fig. (11b) that the flow field is now featured by significant wake flow. The spreading of wake flow can lead to dissipation of energy which lowers energy extraction efficiency. For blade 1, the largest spreading area of wake flow is observed at DU 06-W200 while the smallest one is seen at NACA0015. For blade 2 , which is affected by the wake flow from blade 3, is also found to generate wake flow and disturb blade 1 . The negative influence is more serious at DU 06-W-200 and OPTFoil than NACA0015 and OPTFoil2014. Wake flow generated by NACA0015 and OPTFoil2014 have less blockage effect on the flow field than those generated by DU $06-\mathrm{W}-200$ and OPTFoil, resulting in relatively higher torque coefficient and power coefficient at higher tip speed ratio.

It can be found by the above analysis that the unsteady flow field features varies at different tip speed ratios, influencing the rotor aerodynamic performance. To attain better rotor aerodynamic performance, an $\mathrm{H}$ type VAWT airfoil should strike aerodynamic balance between lower and higher tip speed ratio. Based on the above comparative analysis, only OPTFoil2014 achieves the objective while another three airfoils fail.

\section{CONCLUSION}

(1) Based on the analysis of overall potential of VAWT rotor absorbing effective wind energy resources, a new indicator named averaged effective wind energy utilization coefficient has been defined to evaluate the aerodynamic performance of rotor at variable tip speed ratios and selected as the airfoil design objective.

(2) A mathematic model for direct airfoil design has been constructed on the basis of relatively small amount of CFD calculation by regression design theory. Based on this model, a new airfoil noted as OPTFoil2014 is designed for an $\mathrm{H}$ type VAWT used in urban sites under constant wind speed of $8 \mathrm{~m} / \mathrm{s}$. Compared with NACA0015 which is widely used, the averaged effective wind energy utilization coefficient of rotor with has increased by $6.78 \%$ when OPTFoil2014 is applied on the rotor.

(3) With the changing tip speed ratio, rotor flow field is characterized by different unsteady flow features which have direct influence on aerodynamic performance. Traditional H type VAWT airfoil design method is aiming at aerodynamic performance of single airfoil which is not capable to take the unsteady flow field into account. It is necessary to apply rotor aerodynamic performance at variable tip speed ratios as the airfoil design objective.

\section{CONFLICT OF INTEREST}

The authors confirm that this article content has no conflict of interest.

\section{ACKNOWLEDGEMENTS}

Declared none.

\section{REFERENCES}

[1] W. T. Chong, S. C. Poh, A. Fazlizan, and K.C. Pan, "Vertical axis wind turbine with omni-directional-guide-vane for urban high-rise buildings", J. Cent. South Univ., vol.19, pp. 727-732, 2012.

[2] K. P. You, J. Y. You, and Y. M. Kim, "Wind response of hish-rise building in diversified inlets to construct wind turbine system on roof', J. Cent. South Univ., vol. 21, pp. 4395-4403, 2014.

[3] A. Islam, H. Neveen, and D. Steven, "Effect of roof shape, wind direction, building height and urban configuration on the energy yield and positioning of roof mounted wind turbines", Renew. Energy, vol.50, pp. 1106-1118, 2013.

[4] D. R. Drew, J. F. Barlow, and T. T. Cokeril, "Estimating the potential yield of small wind turbines in urban areas: a case study for greater London UK", J. Wind Eng. Ind. Aerod., vol. 115, pp. 104-111, 2013.

[5] K. Pope, I. Dincer, and G. F. Naterer, "Energy and exergy efficiency comparison of horizontal and vertical axis wind turbines", Renew. Energ., vol. 35, pp. 2102-2113, 2010.

[6] S. Eriksson, H. Bernhoff, and M. Leijon, "Evaluation of different turbine concepts for wind power", Renew. Sust. Energ. Rev., vol. 12, no.5, pp. 1419-1434, 2008.

[7] R. Howell, N. Qin, J. Edwards, and N. Durrani, "Wind tunnel and numerical study of a small vertical axis wind turbine", Renew. Energ., vol. 35, pp. 412-422, 2010.

[8] R. E. Sheldahl, and P, C. Klimas, "Aerodynamic Characteristics of Seven Symmetrical Airfoil Sections Through 180 Degree Angle of Attack for Use in Aerodynamic Analysis of Vertical Axis Wind Turbine", Sandia National Laboratory, Albuquerque, pp. 12-63, 1981.

[9] M. C. Claessen, "The Design and Testing of Airfoils for Application in Small Vertical Axis Wind Turbine", Delft University of Technology, Delft, 2006, pp. 21-90.

[10] M. Islam, D. S. K. Ting, and A. Fartaj, "Design of a special purpose airfoil for smaller capacity straight bladed VAWT", Wind Eng., vol. 31, no.6, pp. 401-424, 2007.

[11] J. J. Qu, Y. Mei, and M. W. Xu, "Optimization of vertical axis wind turbine airfoil design with complex optimum method", Renew. Energy Resour., vol. 29, pp.132-136, 2011 (in Chinese).

[12] C. Shu, X. Cai, P. Pan, J. Zhu, and L.X. Zhang, "Research on aerodynamic and power characteristic for improved airfoil of vertical axis wind turbine", Water Resour. Power, vol. 33, no.1, pp. 162-172, 2015. (in Chinese).

[13] H. A. Yanto, C. T. Lin, J. C. Hwang, and S.C. Lin, "Modeling and control of household-size vertical axis wind turbine and electric power generation system", Proceedings of the International Conference on Power Electronics and Drive Systems, 2009, pp. 1301-1307.

[14] C. Vlad, A. I. Bratcu, I. Munteanu, and S. Epureet, "Real-time replication of a stand-alone wind energy conversion system: Error analysis", Elec. Power Energy Syst., vol. 55, pp. 562-571, 2014.

[15] L. Barote, and C. Marinescu, "Storage analysis for stand-alone wind energy applications", In: Proceedings of the International Conference on Optimization of Electrical and Electronic Equipment, 2010, pp. 1180-1185.

[16] X. M. Chen, and R. K. Agarwal, "Optimization of wind turbine blade airfoils using a multi-objective genetic algorithm", J. Aircraft, vol. 50, no. 2, pp. 519-527, 2013.

[17] R. K. Singha, M. R. Ahmeda, and M. A. Zullahb, "Design of a low Reynolds number airfoil for small horizontal axis wind turbines", Renew. Energ., vol. 42, pp. 66-76, 2012.

[18] F. Scheurich, "Modelling the Aerodynamics of Vertical Axis Wind Turbines", Glasgow: University of Glasgow, 2011, pp.127-129.

[19] M. Islam, A. Fartaj, and R. Carriveau, "Analysis of the design parameters related to a fixed pitch straight bladed vertical axis wind turbine", Wind Eng., vol. 32, no.5, pp. 491-507, 2008. 
[20] L. H. Zhao, M. Liu, and T. Lv, "Study on the aerodynamic performance of blade airfoil of vertical axis wind turbine at low Reynolds number", Inf. Technol. J., vol. 12, no. 14, pp. 3042-3045, 2013.

[21] A. Lida, A. Mizun, and K. Fukudome, "Numerical Simulation of Aerodynamic Noise Radiated from Vertical Axis Wind Turbines", Kogakuin University, Tokyo, pp. 1-4, 2004.

[22] L. A. Danao, N. Qin, and R. Howell, "A numerical study of blade thickness and camber effects on vertical axis wind turbines", $P$. I. Mech. Eng. A-J. Pow., vol. 226, no. 7, pp. 867-881, 2012.

[23] M. R. Castelli, G. Simioni, and E. Benini, "Numerical analysis of the influence of airfoil asymmetry on VAWT performance", Worl Acad. Sci. Eng. Technol., vol. 61, 2012, pp. 312-321.

[24] I. Abbott, and A. V. Doenhoff, "Theory of wing sections including a summary of airfoil data," Dover Publications, New York, 1959, pp. 111-118.
[25] J. O. Rawlings, S. G. Pantula, and D. A. Dickey, "Applied Regression Analysis: A Research Tool, Second Edition,' SpringerVerlag Inc., New York, 1998, pp. 75-149.

[26] F. R. Menter, "Two equation eddy viscosity turbulence models for engineering applications", AIAA J., vol. 32, no. 8, pp. 1598-1605, 1994.

[27] K. M. Almohammadi, D. B. Ingham, L. Ma, and M. Pourkashanian, "2D CFD analysis of the effect of trailing edge shape on the performance of a straight blade vertical axis wind turbine", IEEE Trans. Sustain. Energy, vol. 6, no. 1, pp. 228-234, 2015 .

[28] M. R. Castelli, A. Englaro, and E. Benini, "The Darrieus Wind Turbine: Proposal for a new performance prediction model based on CFD", Energy, vol. 36, pp. 4919-4934, 2011.

[29] O. Gueeri, A. Sakout, and K. Bouhadef, "Simulations of the fluid flow around a rotating vertical axis wind turbine", Wind Eng., vol. 31, no.3, pp. 149-163, 2007.

(C) Yi et al.; Licensee Bentham Open.

This is an open access article licensed under the terms of the (https://creativecommons.org/licenses/by/4.0/legalcode), which permits unrestricted, non-commercial use, distribution and reproduction in any medium, provided the work is properly cited. 